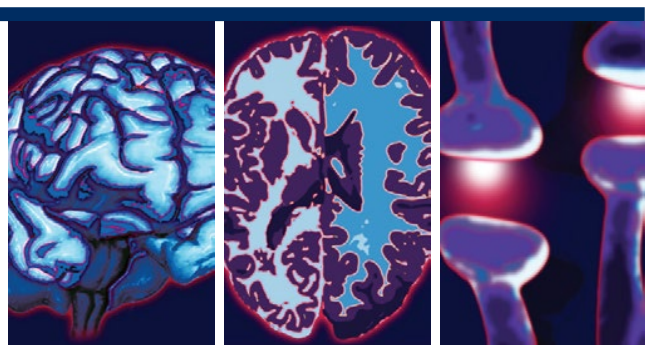

\title{
Factors associated with retention in a methadone maintenance treatment program in heroin-dependent Han Chinese in Taiwan
}

\author{
Jia-Fu Lee ${ }^{1,2, \dagger}$, Shih-Mao Lo ${ }^{3}$, Yun-Hsuan Chang ${ }^{4,5}$, Yue-Cune Chang ${ }^{6}$, Sheng-Yu Lee ${ }^{7}$, Ru-Band Lu ${ }^{5, \dagger}$
}

\begin{abstract}
The longer patients remain in a methadone treatment program, the longer they stay away from drug abuse, and the more counseling and support services they are given, which means that the treatment is more likely to be effective. We analyzed the factors associated with retention in a methadone maintenance treatment in order to predict and improve the retention rate and compliance in heroin-dependent patients. We recruited 356 patients in a methadone maintenance program at a psychiatric hospital in northern Taiwan I. Most participants were male $(n=295,82.86 \%)$, had a troubled marriage $(n=287,80.62 \%)$, had attended only elementary or junior high school $(n=225,63.20 \%)$, and had to pay their own expenses $(n=255,71.62 \%)$. Cox's proportional hazards model was used to evaluate the association of retention rates (up to 700 days) and to analyze the potential risk factors of gender, marital status, education level, payment method, and age. The retention rate was relatively lower for patients who were divorced, who privately paid for treatment, who were younger, and lower educational level. Divorce had the greatest effect (hazard rate ratio $[H R]=2.131 ; \mathrm{p}$-value $=0.002$ ) on the retention rate. More than two-thirds of the subsidized participants persevered with the program, but only half who paid for their own treatment of them persevered $(H R=1.610 ; p=0.017)$. Medical and social workers should keep in mind the various factors that influence retention rates and use them to improve their counseling of drug addicts, as well as to modify the type of social assistance given to methadone maintenance clients. This will help increase the retention rate and improve compliance.
\end{abstract}

\section{Keywords}

Methadone, Heroin, Retention rate

\footnotetext{
'Department of Psychiatry, Taipei Tzu Chi Hospital, Buddhist Tzu Chi Medical Foundation, Taipei, Taiwan

${ }^{2} \mathrm{~S} c h o o l$ of Medicine, Tzu Chi University, Hualien, Taiwan

${ }^{3}$ Department of Psychiatry, Beitou Branch, Tri-Service General Hospital, Taipei, Taiwan

${ }^{4}$ Department of Psychiatry, College of Medicine, National Cheng Kung University, and National Cheng Kung University Hospital, Tainan, Taiwan

${ }^{5}$ Department of Mathematics, Tamkang University, Tamsui, Taiwan

${ }^{6}$ Division of Clinical Psychology, Institute of Allied Health Sciences, College of Medicine, National Cheng Kung University, Tainan, Taiwan

'Department of Psychiatry, Kaohsiung Veterans General Hospital, Kaohsiung, Taiwan
}

${ }^{\dagger}$ Author for correspondence: Jia-Fu Lee, MD, PhD, Department of Psychiatry, Taipei Tzu Chi Hospital, Buddhist Tzu Chi Medical Foundation, 289 Jianguo Road, Xindian Dist., New Taipei City 23142, Taiwan. Tel: 886-6-6628-9779 ext.63780, email: health818@gmail. com

Ru-Band Lu, MD, Institute of Allied Health Sciences, Institute of Behavioral Medicine, Department of Psychiatry, College of Medicine and Hospital, National Cheng Kung University, 138 Sheng-Li Road, Tainan 70403, Taiwan. Tel: 886-6-235-3535 ext. 5108; Fax: 886-6-2095616, email: rblu@mail.ncku.edu.tw, rubandlu@gmail.com 


\section{Introduction}

Methadone, a synthetic opioid, was developed in 1937 and introduced in the United States in 1947 [1]. It has an analgesic effect similar to that of an opioid [2]. Since 1965, methadone has been used to treat heroin addiction and to prevent and improve withdrawal symptoms $[3,4]$. Because it is taken orally, the probability that the user will become infected with diseases such as AIDS, hepatitis B or C, and endocarditis is lower than in those who inject themselves with recreational drugs [5]. In 1985, Australia introduced methadone maintenance treatment programs [6]; furthermore, this type of treatment has also been gradually adopted by European countries since the mid-1990s. In Taiwan, a methadone maintenance treatment program was initiated in February 2006 at six hospitals and clinics. It is now available at 97 medical institutions (75 hospitals and 22 satellite clinics). In total, almost 11,376 drug addicts in Taiwan have participated in methadone maintenance treatment programs (MMTP). These programs have reduced the number of people infected by the HIV virus and thus have significantly helped prevent AIDS among the heroin-dependent population in Taiwan [7-12].

The longer that patients remain in a methadone maintenance treatment program, the longer they stay away from drug abuse, and the more counseling and support services they are given, which means that the treatment is more likely to be effective [10]. Study results vary or even contradict each other on the correlation between short-term treatment and treatment effectiveness. Simpson, et al. [13] reported that there was no significant difference between patients treated for less than 90 days and patients not treated at all. Grella, et al. [14], however, concluded that there was a linear effect of treatment duration on treatment effectiveness.

D'Ippoliti, et al. [15] reported that route of drug use could affect heroin dependents remain in the methadone-maintenance therapy. Their results were consistent with earlier reports about potential risk factors related to retention rate, such as dosage of prescribed methadone [16] , although a flexible dosage policy was reported to be associated with retention rate [17]. In addition, living environment was also reported as potential factor that the quality of counseling and other social support services could affect the treatment outcome [18-20].

Zhang, et al. [21] reported that improvement in primary drug use had a positive linear relationship with treatment retention time among methadone clients, but was not related to the retention threshold. Fareed, et al. [22] reported potential targets for intervention related to lifestyle risk factors and comorbid medical conditions which may improve health outcomes for older opioid dependents. Therefore, investigating the retention rate and the reasons for withdrawal from the treatment program has become an important aspect of whether or not a methadone maintenance treatment program can succeed.

As the heroin addicts' harm reduction behavior and quality of life are associated with MMTP, it is important to maintain long-term support for those who retained in the treatment program. There are several factors affected the maintenance of MMTP such as the medical policy (Medicaid), psychosocial support (gender, marriage) and cognition (age and educational level). Therefore, this study is aimed at explore the effect of MMT program of the basic information on ageEducation level and payment method.

We explored the current status of a methadone maintenance treatment program in a psychiatric hospital in Taiwan to determine what factors significantly affect substance users undergoing methadone maintenance therapy. Moreover, we investigated the potential risk factors that might influence methadone maintenance treatment retention in hopes of establishing a reference and theoretical basis for improving clinical practice.

\section{Methods \\ - Participants}

In this retrospective study, the medical records of 368 patients who had undergone methadone maintenance treatment at a psychiatric hospital (Tri-Service General Hospital Beitou Branch) in Northern Taiwan at least for 2 years (from July, 2007 to July, 2009) were reviewed. All participants before entering the study have been informed and signed the inform consent form. The inclusion criteria were as follows: (1) being more than 20 years old with sufficient cognitive competence to understand and sign an informed consent; (2) meeting the DSM-IV (Diagnostic and statistical manual of mental disorders, fourth edition) criteria for opioid dependence; (3) no other severe medical or mental condition including hepato-encephalopathy, delirium, or behavioral disturbances. At the intake screen, the participants were identified as private expense MMTP or receive subsidy MMTP groups based on whether they met the publicly funded 
eligibility criteria: with HIV seropositive, exprisoners, or applying for deferred prosecution. The definition of being retained is that the patients did not lose follow-up more than 14 consecutive days; while drop out was confirmed by the psychiatrist if patient lost follow up more than 14 consecutive days. Once the patient is meet the definition of drop out, he or she is censored from the dataset and will not be analyzed for further activities. Twelve patients refused to participate in the study after the initial screening interview; a total of 356 (96.7\%) evaluable participants were enrolled. The basic information collected included gender, marital status, educational background, and the method of payment for treatment.

\section{- Statistical analysis}

SPSS 18 for Windows (SPSS, Chicago, IL) was used for all statistical analyses. Descriptive statistics were used to analyze the distribution of the participants' background variables. The Fisher's exact tests and independent t-tests were used to compare the differences of demographic information between the participants who finished the program until the study was completed and those who dropped out. KaplanMeier survival curves and log rank tests were used to compare the retention rates based on gender, educational background, marital status, and payment method. Cox's proportional hazards model was used to compare the retention rates after adjusting for the effects of prognostic factors. Significance was set at $\mathrm{p}<0.05$.

\section{Results}

\section{- Analysis of background variables of the participants undergoing methadone maintenance treatment}

There were far more male than female participants, the male-to-female ratio of opioid-dependent persons was $82.9 \%$ to $17.1 \%$. However, there was no significant difference $(p=0.778)$ in the male-to-female ratio between the participants who were followed-up and those who dropped out (Table 1). Many more participants were single or unmarried and living together than were married or divorced. Moreover, the retention rate for married participants was non-significantly ( $p=0.063$ ) higher than for divorced participants (Table 1). Significantly more participants had an elementary or junior high school education $(63.9 \%)$ than a senior high school or vocational high school (31.5\%), university or higher $(2.4 \%)$.
Two hundred fifty-five (71.6\%) participants paid for their own treatment, and 101 (28.4\%) received a full government subsidy. There was a highly significant $(p=0.005)$ association between the payment method and persevering with the program (Table 1). Specifically, only one-third of the subsidized participants dropped out of the program, but half of those who paid for their own treatment dropped out.

\section{- Differences in retention rate between participants with different backgrounds}

\section{Differences based on payment method}

A Kaplan-Meier survival curve shows that the retention rate of participants with both payment methods rapidly decreased from the start of treatment (Figure 1). After approximately 400 days, the retention rates more or less stagnated and did not decrease. After day 200, the retention rate of the subsidized participants was still greater than $60 \%$, but the retention rate of participants who used their own money was only $40 \%$. A logrank test showed a significant difference between those two survival curves $(\mathrm{p}=0.007)$.

\section{Differences based on gender}

The retention rate curves for men and women follow a path similar to that of the payment curves: an initial drop-off followed by stabilization (Figure 2). However, the curve showed that the decreased maintenance retention rate for women flattened on treatment day 150, but that for men, it did not flatten until treatment day 250. On treatment day 200, the retention rate for women was approximately $55 \%$, but for men it was only $45 \%$, a non-significant difference $(\mathrm{p}=0.311)$.

\section{Differences based on marital status}

The retention rate was highest for married persons and lowest for divorced persons (Figure 3). On treatment day 200, the retention rate was approximately $60 \%$ for married persons, $50 \%$ for single persons and for those who were unmarried and living together and only $25 \%$ for divorced persons. A log-rank test showed a highly significant difference between these three groups $(\mathrm{p}=0.003)$.

\section{Differences based on education level}

All three Kaplan-Meier survival curves for education level showed an initial drop followed by relative equilibrium (Figure 4). The curve for participants educated to elementary school and junior high school levels reached equilibrium at approximately $39 \%$ on about treatment day 300 , 
Factors associated with retention in a methadone maintenance treatment program in heroindependent Han Chinese in Taiwan

\section{Research}

but the curve for participants educated to senior high school (academic education in Taiwan) or vocational high school level reached equilibrium at approximately $56 \%$ on about treatment day 200. The number of participants with a university or higher $(n=16,4.5 \%)$ was relatively small, and thus the curve representing these participants first showed a sharp downtrend that stabilized at approximately $24 \%$ on about treatment day 210. The overall differences between these three groups were significant ( $\mathrm{p}=0.029)$.

\section{- Models assessing the factors influencing the methadone maintenance treatment program retention rate}

We used Cox's proportional hazards model to explore the potential risk factors of the retention rate. In the model, the possible covariates were gender, marital status, education level, payment method, and age. After an adjustment for the effects of other factors presented in Table 2, the risk of instantaneously quitting treatment (hazard rate) for divorced participants was $113.1 \%$ significantly higher than that for single or living together participants (hazard rate ratio $[H R]=2.131$; $\mathrm{p}$-value $=0.002$ ), but for married participants, it was $11.7 \%$ nonsignificantly lower than that for single or living together participants $(\mathrm{HR}=0.883 ; \mathrm{p}=0.57)$. Similarly, the risk of instantaneously quitting treatment for participants with a senior or vocational high school education was $60.6 \%$ significantly higher than that for participants with university or higher $(\mathrm{HR}=1.606, \mathrm{p}=0.012)$. The risk of instantaneously quitting treatment was significantly higher for participants who used their own money than that for those who received a government subsidy $(\mathrm{HR}=1.610$; $\mathrm{p}=0.017)$, and lower (2.8\% lower per year) for older than for younger participants $(\mathrm{HR}=0.972$; $\mathrm{p}=0.005)$. However, the risk of instantaneously quitting treatment based on gender was not significant $(\mathrm{p}=0.431)$.

\section{Discussion}

This study was to evaluate the risk factors affecting retention in a 700-day methadone maintenance therapy program. There were far more male $(82.9 \%)$ than female $(17.1 \%)$ participants. This ratio reflects similar information from a cohort of prisoners who underwent methadone maintenance treatment in Taiwan. In that study, the male-to-female ratio of opioid-dependent persons was $88 \%$ to $12 \%$ [23]. There were significantly more men than women were in the
Table 1: Comparison of the Differences of the Demographic Information between the Persevered and Withdrawal Subjects in the Methadone Maintenance Program $(\mathrm{N}=356)$.

\begin{tabular}{|l|l|l|l|l|}
\hline & Variable & $\begin{array}{l}\text { Persevere with } \\
\text { the Program }\end{array}$ & $\begin{array}{l}\text { Withdraw from } \\
\text { the Program }\end{array}$ & p-value \\
\hline Sex (Number, \%) & Male & $162(54.9 \%)$ & $133(45.1 \%)$ & 0.778 \\
\hline & Female & $35(57.4 \%)$ & $26(42.6 \%)$ & \\
\hline $\begin{array}{l}\text { Marital Status } \\
\text { (Number, \%) }\end{array}$ & Married & $42(60.9 \%)$ & $27(39.1 \%)$ & 0.063 \\
\hline & Divorced & $13(37.1 \%)$ & $22(62.9 \%)$ & \\
\hline & Others & $142(56.3 \%)$ & $110(43.7 \%)$ & \\
\hline Education ${ }^{\mathrm{b}}$ (Number, \%) & Group 1 & $112(49.8 \%)$ & $113(50.2 \%)$ & 0.020 \\
\hline & Group 2 & $74(64.3 \%)$ & $41(35.7 \%)$ & \\
\hline & Group 3 & $11(68.8 \%)$ & $5(31.3 \%)$ & \\
\hline $\begin{array}{l}\text { Payment method (Number, } \\
\%)\end{array}$ & Private Expense & $129(50.6 \%)$ & $126(49.4 \%)$ & 0.005 \\
\hline $\begin{array}{l}\text { Initial dose (mean } \pm \text { SD mg ) } \\
\text { Receive subsidy }\end{array}$ & $68(67.3 \%)$ & $33(32.7 \%)$ & \\
\hline $\begin{array}{l}\text { Last dose (mean } \pm \text { SD mg ) } \\
\text { Numbers of prescription }\end{array}$ & $29.06 \pm 12.76$ & $30.10 \pm 12.29$ & $0.44^{c}$ \\
\hline $\begin{array}{l}\text { a: Others: Single or living together } \\
\text { b: Group 1: Elementary or Junior High School; Group 2: Senior or Vocational High School; Group } \\
\text { 3: Others(University or higher) } \\
\text { c: Independent t test } \\
\text { Initial dose: the 1st day methadone administer dose. } \\
\text { Last dose: the final methadone maintained dose. }\end{array}$ & $48.71 \pm 25.12$ & $55.41 \pm 28.13$ & $<0.0005^{c}$ \\
\hline
\end{tabular}

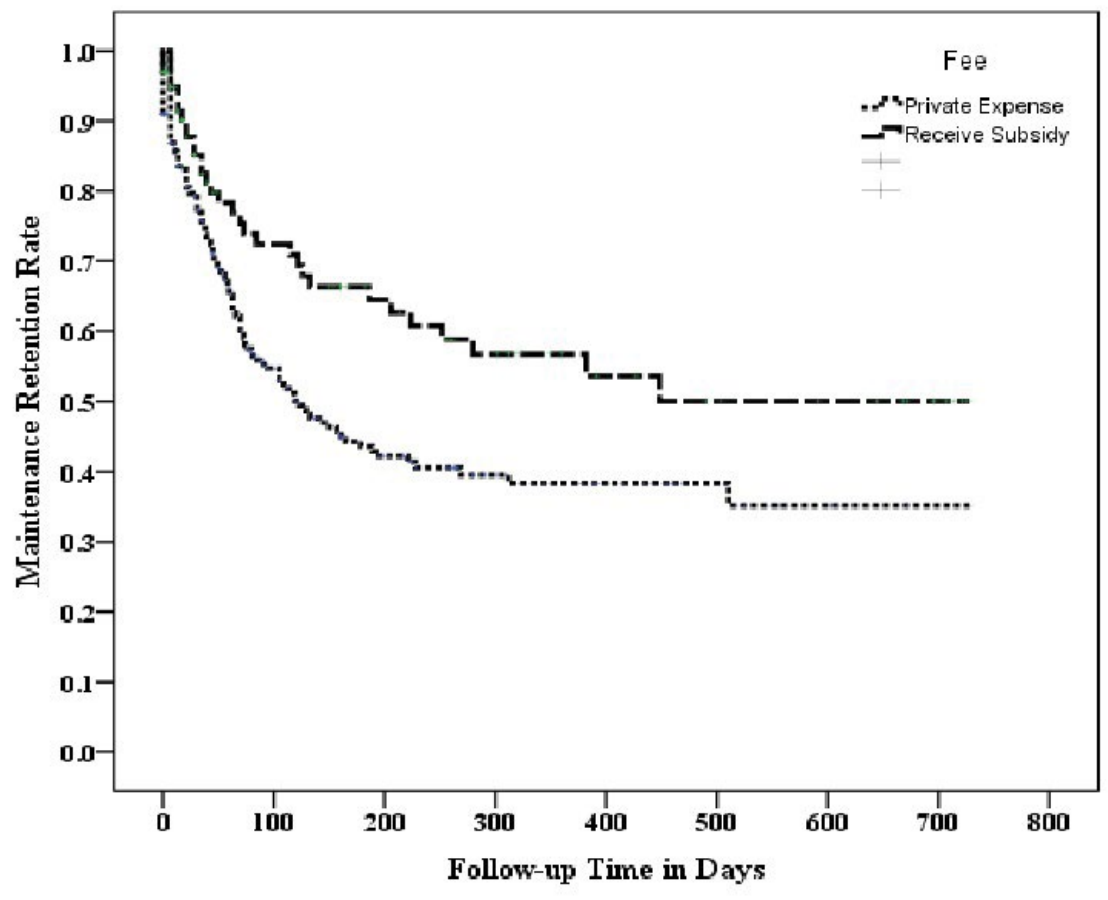

Figure 1: Methadone Maintenance Retention Rate by Payment Method (unadjusted estimates) with $p=0.007$ (Log Rank test).

methadone maintenance treatment program. Although men have historically been more likely to seek treatment for heroin use, the rate of women seeking treatment has increased in recent decades. By 2010, there were nearly equal 


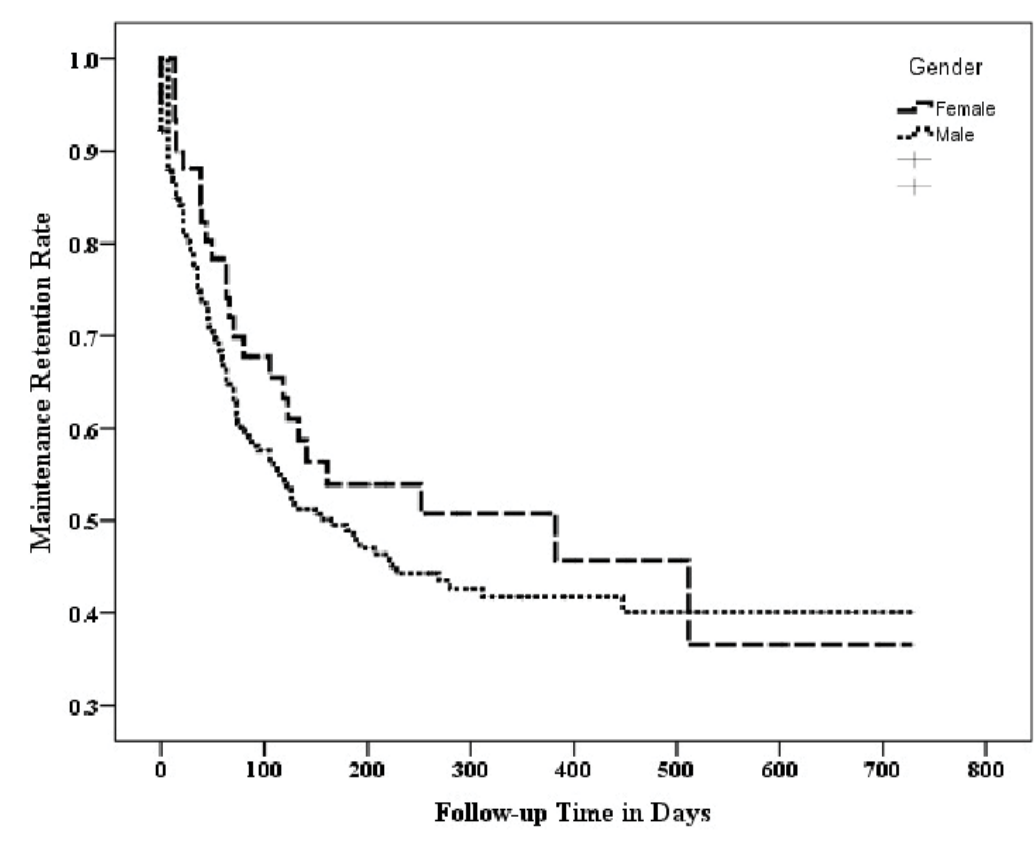

Figure 2: Methadone Maintenance Retention Rate by Gender (unadjusted estimates) with $\mathrm{p}=0.311$ (Log Rank test).

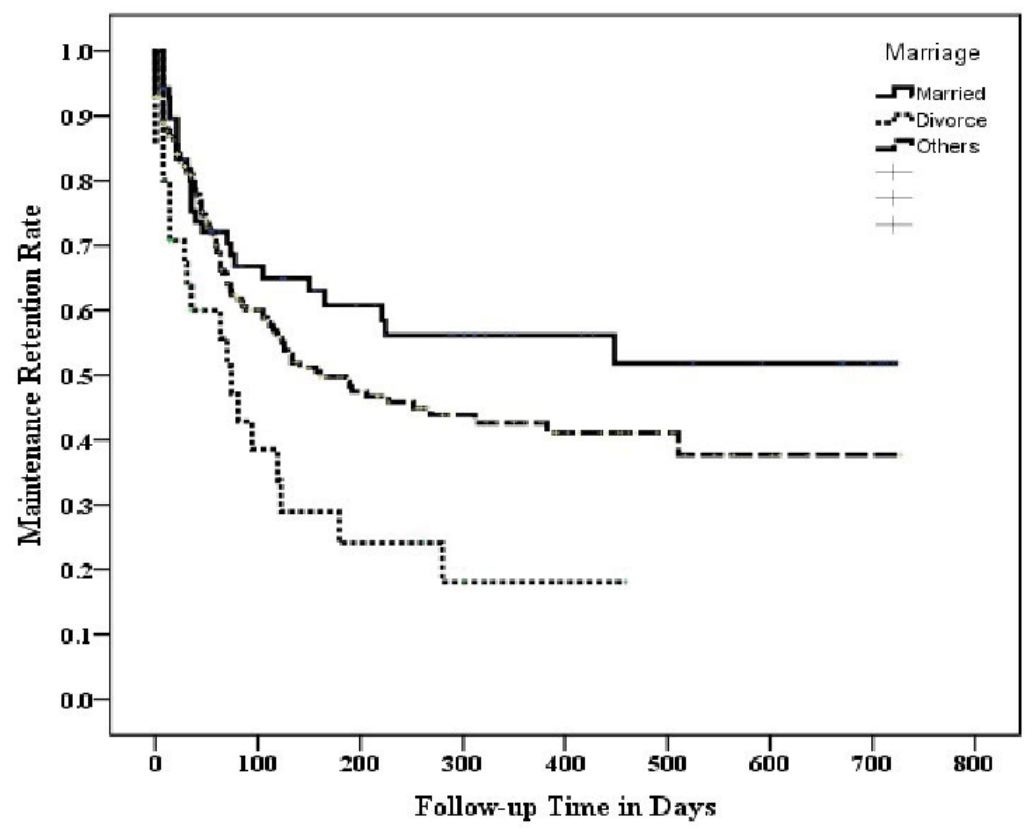

Figure 3: Methadone Maintenance Retention Rate by Marital Status (unadjusted estimates) with $p=0.003$ (Log Rank test). Others: Single or unmarried and living together.

numbers of male and female heroin users seeking treatment [24]. In addition, our results, which showed no significant gender-based difference in retention rates, differ from the studies as Tang [23] and Newman [25]. Tang, et al. [23] found a correlation between gender and retention rate, and that the retention rate for females was relatively higher. Newman, et al. [25] found that treatment retention time was relatively longer in women who were older than 30 .

Overall, the education level of the participants was generally relatively low: 63.2\% (225/356) had only an elementary or junior high school education. The differences in the retention rate on treatment day 200 were approximately $60 \%$ (69/115) for those with a senior high school or vocational high school education versus approximately $40 \%(90 / 225)$ for those with an elementary school or junior high school education. Both, however, were higher than the approximately $20 \%(3 / 16)$ for those with a university or higher education. These results are similar to the results by Tang, et al. [23], who found [a] a correlation between education level and retention rate, and $[b]$ a higher retention rate for those with a senior high school or vocational high school education than for those with an elementary school or junior high school education. In our study, the number of participants with a university or higher education was too small $(16 / 356=4.5 \%)$ to allow us to draw any conclusions about their retention rate.

Previous studies [13] suggest that being married is associated with a reduction in antisocial behavior (e.g., substance abuse) and that being married is associated with lower crime rates. Another report [26] also supports the inference that marriage casually inhibits crime in the course of a lifetime. We found that our relatively stable married participants were significantly fewer in number than our single and divorced participants. This may indicate that family plays an important role in the onset and development of heroin abuse as well as in its prevention and in the recovery process. If the spouse of a heroindependent patient is unable to offer sufficient encouragement and support because he or she lacks concern for, understanding of, and confidence in the patient, the patient will tend to withdraw and become mentally distressed, indifferent, and irritable. This will eventually result in the patient's leaving the treatment program and probably taking heroin again. These findings are similar to those of other studies as Tang, et al. [23] and Simpson [13]. They found a positive correlation between being married and retention rate [26], and a negative correlation between the retention rate and being divorced or widowed [13].

Most patients were paying their own treatment expenses because there is little government support for this type of program, and what 
little support is available is difficult to obtain. We found that the retention rate was higher for government-subsidized patients, which implies that the cost of treatment is an important factor that affects both treatment participation and retention. This finding is similar to those of other studies. Kwiatkowski, et al. [27] found that subsidized treatment significantly prolonged retention time, and, that even if they had never undergone or had previously been unwilling to undergo treatment, when the subsidized treatment was no longer provided, many still continued with the program. Booth, et al. [3] found that patients given subsidized treatment for 90 days were 4.63 times more likely to remain in the treatment program than were patients over the same period not given subsidized treatment. In addition, in our study, even after 4-6 months of treatment, the retention rate for patients given subsidized treatment was significantly higher than for those not not given subsidized treatment. The others, the patients who have government support were fulfilled certain criteria: with HIV seropositive, ex-prisoners, or applying for deferred prosecution may also affcted the retention rate between self-funded and publicly-funded MMT.

Our study used only gender, education level, marital status, and payment method to analyze retention rate. However, the results from Tang, et al. [23] and Deng, et al. [6] indicate that various other factors, such as physiology, psychology, environment, health status, possible legal problems, and the severity of addictionrelated problems, also affect the retention rate. Furthermore, other variables, namely, genetics, spirituality (religious belief), and the methadone dose, appear to affect adherence. We hope that future studies will be able to analyze these factors so that a more accurate and realistic prediction model can be established. Such a model will improve our understanding of why methadone maintenance treatment retention rates vary, and will also serve as a reference for psychiatric clinicians so that perhaps retention rates and compliance can be increased.

From application indication, we would provide evidence to suggest that psycho-social factors would influence the MMT retention rate. For marriage, we found that divorce or unstable marriage was a risk factor for low MMT retention rate. This would remind those clinicians and workers to further give more psychological and social support to those participants who are in unstable marriage or divorce. In addition, we

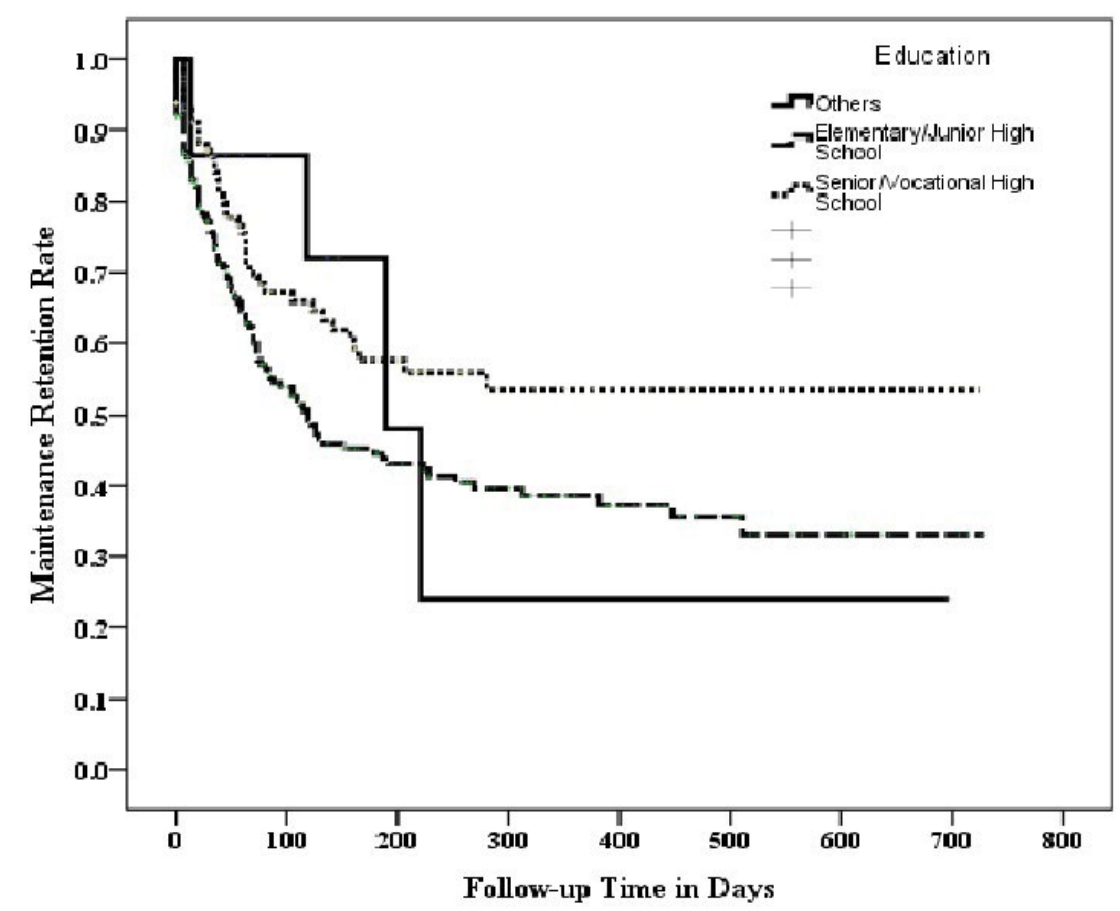

Figure 4: Methadone Maintenance Retention Rate by Educational Level (unadjusted estimates) with $p=0.025$ (Log Rank test). Others (University or higher).

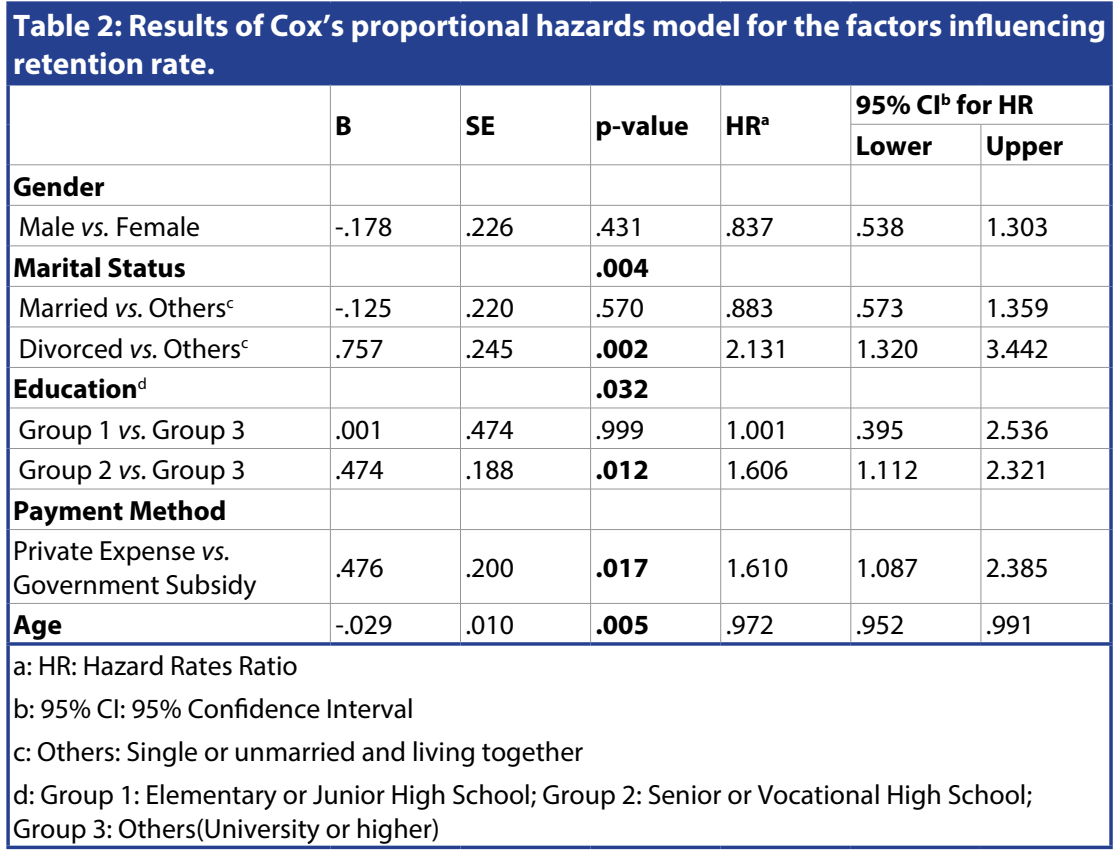

would suggest that our government to keep this welfare because the payment was shown as an important factor to keep these participants in the treatment program. Moreover, educational level was another risk factor, although it might be hard for those participants go back to school. Previous studies have been proved that cognitive therapy would change addiction behavior; giving 
psycho-education would help these participants to improve their cognition and knowledge of addiction.

Because of limited personnel, material resources, and time, this study was done using patients from only one psychiatric hospital in Taipei. The consequence of interpreting our results is when the participants were only from one psychiatric hospital in Taipei. In addition, some potential risk factors were not included, such as living area and methadone dose during treatment [15], although this factor was reported as having less potential than others for causing patients to quit treatment [28]. Moreover, sharing needles is another unconsidered risk for patients in the present study. The results may therefore not be applicable or extendable to other medical institutions. We suggest that future studies recruit more participants and include patients from multiple hospitals and various regions in Taiwan. We also suggest that a parallel qualitative study (interviews, case studies, and didactic analysis) provide a more comprehensive clinical picture. Patients who have a long history of drug use and who have a stable income were more likely to stay in methadone maintenance treatment. Independent of patient characteristics, retention improved dramatically in the first years of programmed functioning, suggesting that patient retention is a sensitive indicator of programmed performance.

\section{Conclusion}

The methadone maintenance treatment retention rate is an important index because it measures the effectiveness of treatment. The longer patients can be retained in the program, the longer they can stay away from drug abuse. Prolonging their stay in the program provides additional opportunities to offer patients more counseling and support, which also tends to make the treatment more effective. Our findings indicate that there are many factors, including marital status, education level, payment method, and age, that influence treatment retention rates. Logistic regression analysis showed that the retention rates for patients who were divorced, paid their own expenses, and were younger were relatively low. Nonetheless, based on the present study, we believe that medical and social workers need to provide additional counseling and social support that targets younger, financially disadvantaged, and unmarried (single, divorced) patients in order to increase retention rates. Such an approach should reduce the dangers of heroin dependence and the spread of various diseases related to drug abuse.

\section{References}

1. Payte JT. A brief history of methadone in the treatment of opioid dependence: a personal perspective. J. Psychoact. Drugs 23(2), 103-107 (1991)

2. Bell J, Burrell T, Indig D, et al. Cycling in and out of treatment; participation in methadone treatment in NSW, 1990-2002. Drug. Alcohol. Depend 81(1), 55-61 (2006).

3. Booth RE, Corsi KF, Mikulich-Gilbertson SK. Factors associated with methadone maintenance treatment retention among street-recruited injection drug users. Drug. Alcohol. Depend 74(2), 177-185 (2004).

4. Burt SA, Donnellan MB, Humbad MN, et al. Does marriage inhibit antisocial behavior? An examination of selection vs causation via a longitudinal twin design. Arch. Gen. Psychiatry 67(12), 1309-1315 (2010).

5. Davstad I, Stenbacka M, Leifman A, et al. Patterns of illicit drug use and retention in a methadone program: a longitudinal study. J. Opioid. Manag 3(1), 27-34 (2007).

6. Deng C, Ma X, Zhou H, et al. Quality of life of heroin dependent patients with methadone maintenance therapy. J.
Sichuan University Medical Science Edition 40(1), 539-543 (2009)

7. Chang KC, Lin CY. Effects of publicly funded and quality of life on attendance rate among methadone maintenance treatment patients in Taiwan: an 18-month follow-up study. Harm. Reduct. J 12(1), 40 (2015).

8. Lin CY, Chang KC, Wang JD, et al. Quality of life and its determinants for heroin addicts receiving a methadone maintenance program: Comparison with matched referents from the general population. J. Formos. Med. Assoc 115(9), 714-727 (2016).

9. Wang PW, Wu HC, Yen CN, et al. Change in quality of life and its predictors in heroin users receiving methadone maintenance treatment in Taiwan: an 18-month followup study. Am. J. Drug. Alcohol. Abuse 38 (2012).

10. Lee TS, Shen HC, Wu WH, et al. Clinical characteristics and risk behavior as a function of HIV status among heroin users enrolled in methadone treatment in northern Taiwan. Subst. Abuse. Treat. Prev Policy 6 (2011).

11. Lin SH, Chen KC, Lee SY, et al. The economic cost of heroin dependency and quality of life among heroin users in Taiwan. Psych. Res 209(3), 512-527 (2013).

12. Yen CN, Wang CS, Wang TY, et al. Quality of life and its correlates among heroin users in Taiwan. The Kaohsiung J. Med. Sci 27(5):17783 (2011).

13. Simpson DD, Joe GW, Rowan-Szal GA, Greener JM. Drug abuse treatment process components that improve retention. J. Subst. Abuse. Treat 14(6), 565-572 (1997).

14. Grella CE, Hser YI, Joshi V, et al. Patient histories, retention, and outcome models for younger and older adults in DATOS. Drug. Alcohol. Depend 57(2), 151-166 (1999).

15. D'Ippoliti D, Davoli M, Perucci CA, et al. Retention in treatment of heroin users in Italy: the role of treatment type and of methadone maintenance dosage. Drug. Alcohol. Depend 52(2), 167-171 (1998).

16. Drummer $\mathrm{OH}$, Syrjanen $\mathrm{M}$, Opeskin $\mathrm{K}$, et al. Deaths of heroin addicts starting on a methadone maintenance programme. Lancet 335(8681), 108 (1990).

17. Brown BS, Watters JK, Iglehart AS. Methadone maintenance dosage levels and program retention. Am. J. Drug. Alcohol. Abuse 9(2), 129-139 (1982). 
Factors associated with retention in a methadone maintenance treatment program in heroindependent Han Chinese in Taiwan

18. Condelli WS, Dunteman GH. Exposure to methadone programs and heroin use. Am. J. Drug. Alcohol. Abuse 19(1), 65-78 (1993).

19. McLellan At, Arndt IO, Metzger DS, et al. The Effects of Psychosocial Services in Substance Abuse Treatment. J. Addict. Nursing 38-47 (1993).

20. McLellan AT, Woody GE, Luborsky L, et al. Is the counselor an "active ingredient" in substance abuse rehabilitation? An examination of treatment success among four counselors. J. Nervous. Mental. Disease 176(7), 423-430 (1988).

21. Zhang Z, Friedmann PD, Gerstein DR. Does retention matter? Treatment duration and improvement in drug use. Addiction 98(5),
673-684 (2003)

22. Fareed A, Casarella J, Amar R, et al. Benefits of Retention in Methadone Maintenance and Chronic Medical Conditions as Risk Factors for Premature Death Among Older Heroin Addicts. J. Psych. Pract 15(3), 227-234 (2009).

23. Tang $\mathrm{H}$, Chang $\mathrm{K}$, Cheen $\mathrm{P}$, et al. Exploring the factors affecting retention rate on methadone maintenance treatment and the intervention strategies. Ann. Meet. Conf. Taiwan. Soc. Addict Sci (2009).

24. Cicero TJ, Ellis MS, Surratt HL, et al. The changing face of heroin use in the united states: A retrospective analysis of the past 50 years. JAMA. Psych 71(7), 821-826 (2014).

25. Newman RG, Tytun A, Bashkow S. Retention of patients in the New York City methadone maintenance treatment program. Int. J. Addict 11(5), 905-931 (1976)

26. Caplehorn JRM, Dalton MSYN, Cluff MC, et al. Retention in methadone maintenance and heroin addicts' risk of death. Addiction 89(2), 203-207 (1994).

27. Kwiatkowski CF, Booth RE, Lloyd LV. The effects of offering free treatment to streetrecruited opioid injectors. Addiction 95(5), 697-704 (2000).

28. Del Rio M, Mino A, Perneger TV. Predictors of patient retention in a newly established methadone maintenance treatment programme. Addiction 92(10), 1353-1360 (1997). 